\title{
Slanted Tri-gates for High-Voltage GaN Power Devices
}

\author{
Jun Ma and Elison Matioli, Member, IEEE
}

\begin{abstract}
In this work we introduce and demonstrate the concept of slanted tri-gates to enhance the breakdown voltage $\left(V_{\mathrm{BR}}\right)$ in lateral GaN power devices. Conventionally field plates (FPs) are used to enhance the $V_{\mathrm{BR}}$ by distributing more homogeneously the electric field near the gate electrode, which is mainly determined by their pinch-off voltage $\left(V_{\mathrm{p}}\right)$. These FPs however rely on a vertical approach, in which $V_{\mathrm{p}}$ is usually designed via the thickness of the FP oxide. On the other hand, the slanted tri-gate relies on a lateral design to tailor its $V_{\mathrm{p}}$, by simply changing the width $(w)$ of their nanowires lithographically. Here, we demonstrate this concept for AIGaN/GaN-on-silicon MOSHEMTs resulting in an increase of $\sim 500 \mathrm{~V}$ in $V_{\mathrm{BR}}$ compared to the counterpart planar devices. These devices presented a high $V_{\mathrm{BR}}$ of $1350 \mathrm{~V}$ with a small gate-to-drain separation $\left(L_{G D}\right)$ of $10 \mu \mathrm{m}$, along with a record high-power figure-of-merit (FOM) of $1.2 \mathrm{GW} / \mathrm{cm}^{2}$ among GaN-on-silicon lateral transistors.
\end{abstract}

Index Terms-GaN, HEMT, tri-gate, slanted tri-gate, field plate, breakdown.

\section{INTRODUCTION}

$\mathrm{L}$ ateral GaN devices are very promising for future power applications, offering large critical breakdown field, high saturation electron velocity and high-mobility 2-dimensional electron gas (2DEG). A current major challenge is the limited voltage-blocking performance in these devices, which is still far from the GaN materials capabilities. An important reason for such early breakdown is the inhomogeneous distribution of the electric field. When a high voltage is blocked in OFF state, the electric field concentrates at the edge of the gate electrode, leading to the early breakdown of the device [1]-[5]. To spread more homogeneously the electric field, various designs of FPs have been developed [4]-[6], among which slant FPs have been proven more effective [7]-[14]. Such slant FPs are achieved by a precise control over the thickness and angle of the sloped oxide in the vertical direction, which is however extremely challenging and difficult to control, and limits the design flexibility of the FPs. More importantly, a large $L_{\mathrm{GD}}$ is still needed for high-voltage blocking, which degrades significantly the on-resistance $\left(R_{\mathrm{ON}}\right)$ of the device [15].

This work was supported in part by the European Research Council under the European Union's H2020 program/ERC grant Agreement 679425 and in part by the Swiss National Science Foundation under Assistant Professor (AP) Energy Grant PYAPP2_166901

The authors are with the Power and Wide-band-gap Electronics Research Laboratory (POWERlab), École polytechnique fédérale de Lausanne (EPFL), CH-1015 Lausanne, Switzerland. (e-mail: jun.ma@epfl.ch; elison.matioli@epfl.ch).

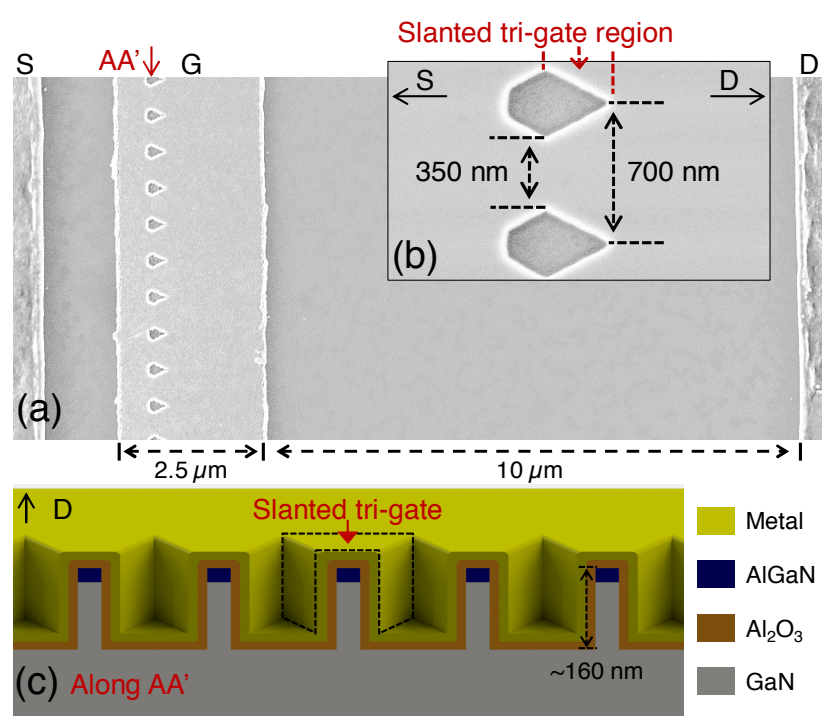

Fig. 1. Top-view SEM images of the slanted tri-gate MOSHEMT (a) with and (b) without the gate dielectric and the metal. (c) A cross-sectional schematic of the slanted tri-gate along the arrow AA'.

In this work, we present a novel concept of slanted tri-gate architecture which, similarly to vertical slant FPs, spreads more effectively the electric field and improves greatly the $V_{\mathrm{BR}}$ of lateral power devices. Different from vertical slant FPs, slanted tri-gate can be easily and accurately engineered with a lateral approach, by varying lithographically the width of the tri-gate nanowires. We demonstrate this concept of slanted tri-gates in GaN MOSHEMTs on silicon substrates, as shown in Fig. 1. Compared with counterpart planar transistors, the $V_{\mathrm{BR}}$ was enhanced by nearly $500 \mathrm{~V}$ using the slanted tri-gate, reaching the buffer limit of $\sim 1350 \mathrm{~V}$ at $1 \mu \mathrm{A} / \mathrm{mm}$ with a $L_{\mathrm{GD}}$ as small as $10 \mu \mathrm{m}$, and rendering a state-of-the-art FOM up to 1.2 $\mathrm{GW} / \mathrm{cm}^{2}$.

\section{PRINCIPLE AND METHODOLOGY}

To explain our concept, let us first present the general working principle of FPs. The most important design variable for FPs is their pinch-off voltage $\left(V_{\mathrm{p}}\right)$, which effectively controls the distribution of the potential $(\Phi)$ and the electric field $(E)$ in a lateral device. Figure 2 shows a simplified model on the effect of the $V_{\mathrm{p}}$ in spreading the electric field within different FP designs [1]-[3]. For devices without the FP (Fig. $2(\mathrm{a})), \Phi$ in the channel increases sharply to the drain voltage $V_{\mathrm{D}}$ at the edge of the gate, where the $E$ peaks. There is nearly no voltage drop between the gate and the drain due to the

(C) 2017 IEEE. Personal use of this material is permitted. Permission from IEEE must be obtained for all other uses, in any current or future media, including reprinting/republishing this material for advertising or promotional purposes, creating new collective works, for resale or redistribution to servers or lists, or reuse of any copyrighted component of this work in other works. 
(a)
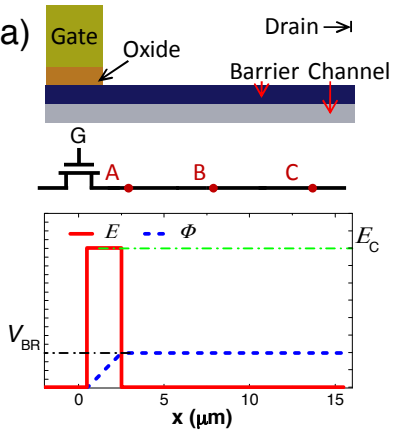

(C)
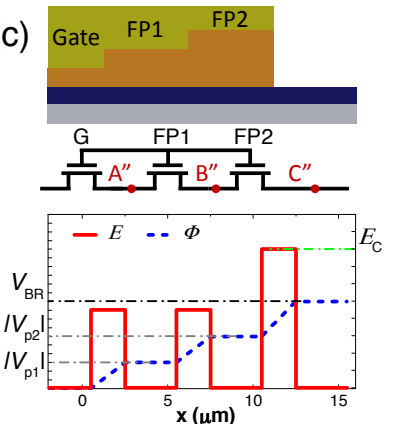

(b)

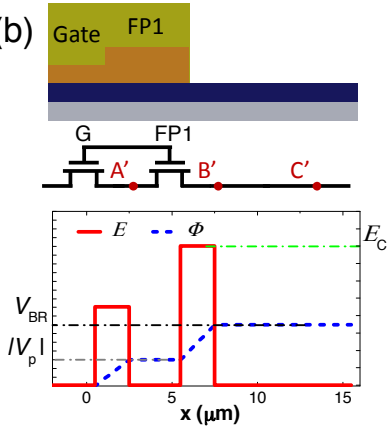

(d)

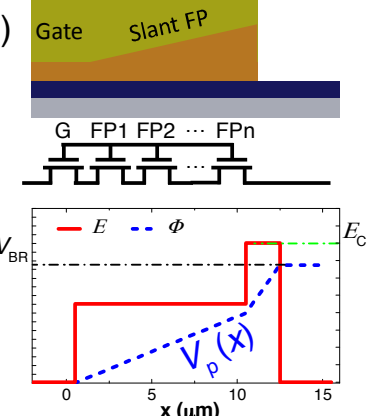

Fig. 2. Schematics, equivalent circuits and distributions of potential $(\Phi)$ and electric field $(E)$ in lateral GaN transistors in OFF state with (a) no FPs, (b) a single FP, (c) two FPs and (d) a slant FP.

high-conductivity $2 \mathrm{DEG}$ channel, thus $\mathrm{E} \sim 0$. When $E$ reaches the critical breakdown field $\left(E_{\mathrm{C}}\right)$, the devices breaks and the $V_{\mathrm{BR}}$ is determined from the integral of the $E(x)$. A FP operates as a transistor in series with the gate (Fig. 2(b)), with a more negative $V_{\mathrm{p}}$ due to the thicker oxide in the FP region. When the FP pinches off the channel underneath, the $\Phi$ at A' is fixed at a certain value (about $\left|V_{\mathrm{p}}\right|$ in a simplified model according to Refs. [1] and [2]). This creates a two-step distribution of $\Phi$, thus the total $E$ is shared between two peaks at the edges of both the gate and the FP. The device breaks when either of the two peaks reaches $E_{\mathrm{C}}$, which results in a larger voltage as compared to the device without the FP due to the larger integral of $E(x)$ spread in two peaks. Since a single FP is insufficient to block large voltages, multiple FPs are usually adopted to distribute better the field. As shown in Fig. 2(c), multiple FPs introduce more equivalent transistors with different $V_{\mathrm{p}}$, creating more steps in $\Phi$ and hence more distributed peaks in $E$. The ultimate outcome of increasing the number of FPs is a slant FP (Fig. 2(d)), which functions as many incrementally-stepped FPs, offering a continuous gradient of $V_{\mathrm{p}}$ towards the drain. This distributes $\Phi$ across the FP as a function of $x$, spreads continuously the $E$ along the entire FP region and thus improves the $V_{\mathrm{BR}}$.

Therefore, the gradient of the $V_{\mathrm{p}}$ is crucial to spread the field and enhance the $V_{\mathrm{BR}}$. Conventionally such gradient is obtained by a sloped etching of the oxide in a slant FP, which is however complex and difficult to design and control, restricting the realization of optimized FP designs. In this work we propose to achieve this gradient of $V_{\mathrm{p}}$ using a tri-gate structure in which $V_{\mathrm{p}}$ can be easily tailored in a simple fabrication process by varying the width of the nanowire $(w)$ composing the tri-gate, without the laborious engineering of the thickness of the oxide. As shown in Fig. 3(a) and (b), the $V_{\mathrm{p}}$ increases smoothly with narrowing nanowires. This is mainly due to the partial relaxation of the $\mathrm{AlGaN} / \mathrm{GaN}$ nanowires as well as additional electrostatic control from the sidewall gates (for the range of $w$

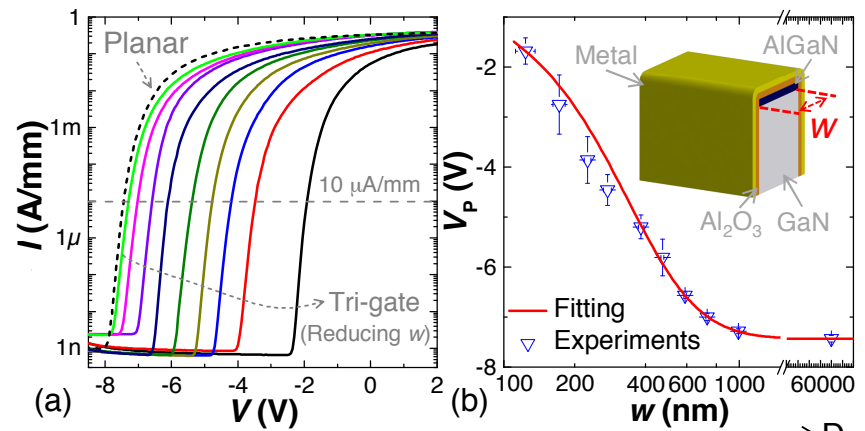

(c)
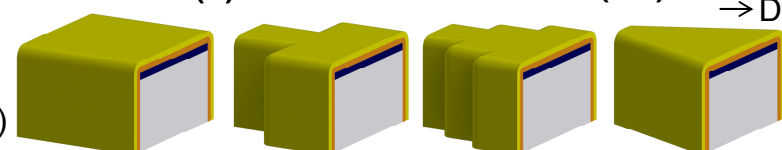

Fig. 3. (a) Average pinch-off characteristics of tri-gate $\mathrm{AlGaN} / \mathrm{GaN}$ structures with different nanowire widths $(w)$. (b) Dependence of the pinch-off voltage $\left(V_{\mathrm{p}}\right)$ on the $w$, in which the error bars were determined from about eight devices of each kind. The inset shows a schematic of a tri-gate AlGaN/GaN structure. (c) Schematics showing the evolution from a single tri-gate, to multiple tri-gates, and to a slanted tri-gate to engineer the gradient of the $V_{\mathrm{P}}$ with $w$.

investigated in this work) $[16,17]$. The smooth dependence of $V_{\mathrm{p}}$ on $w$ offers a much more controllable way to obtain the gradient of the $V_{\mathrm{p}}$ with the tri-gate architecture. As shown in Fig. $3(\mathrm{c})$, a single tri-gate, as we demonstrated recently in Refs. $[16,18]$, can easily evolve into multiple tri-gates, and ultimately into a slanted tri-gate, by simply tuning the $w$ via lithography.

\section{DEVICE FABRICATION}

To demonstrate this concept, we fabricated slanted tri-gate AlGaN/GaN MOSHEMTs on silicon (Fig. 1), in which the $\mathrm{AlGaN} / \mathrm{GaN}$ nanowires were defined with a slanted width and etched with a depth of $\sim 160 \mathrm{~nm}$. The $w$ of the slanted nanowire varied from $350 \mathrm{~nm}$ at its source side to $700 \mathrm{~nm}$ at its drain side (Fig. 1(b)). $20 \mathrm{~nm}$ of $\mathrm{Al}_{2} \mathrm{O}_{3}$ was deposited by atomic layer deposition as gate dielectric, followed by gate metallization. As shown in Fig. 1(c), the oxide and the gate metal in the slanted tri-gate region wrap around the slanted nanowires. The source-side and drain-side planar portions of the gate metal were $0.5 \mu \mathrm{m}$ - and $1.5 \mu \mathrm{m}$-long, respectively. We also fabricated MOSHEMTs with conventional planar gates and tri-gates on the same chip for comparison. They shared the same design and dimensions as the slanted tri-gate device, except for the nanowires. The planar device had no nanowires, while the tri-gate device had a constant $w$ of $600 \mathrm{~nm}$, instead of slanted. The drain-side planar portion of the gate metal in the tri-gate device was $1.3 \mu \mathrm{m}$-long, which is slightly smaller than in the slanted tri-gate device but does not cause any significant changes in the $V_{\mathrm{BR}}$ according to our observation.

All device characteristics in this work, such as drain current $\left(I_{\mathrm{D}}\right)$, transconductance $\left(g_{\mathrm{m}}\right)$ and OFF-state leakage current $\left(I_{\mathrm{OFF}}\right)$, were normalized by the width of the device footprint $(60$ $\mu \mathrm{m})$. To understand the isolated effect of slanted tri-gate in enhancing the $V_{\mathrm{BR}}$, we did not adopt conventional FPs or passivation in these devices.

\section{RESULTS AND DISCUSSION}

To understand the enhancement in $V_{\mathrm{BR}}$ by the slanted tri-gate, we compare the breakdown characteristics the planar, tri-gate 

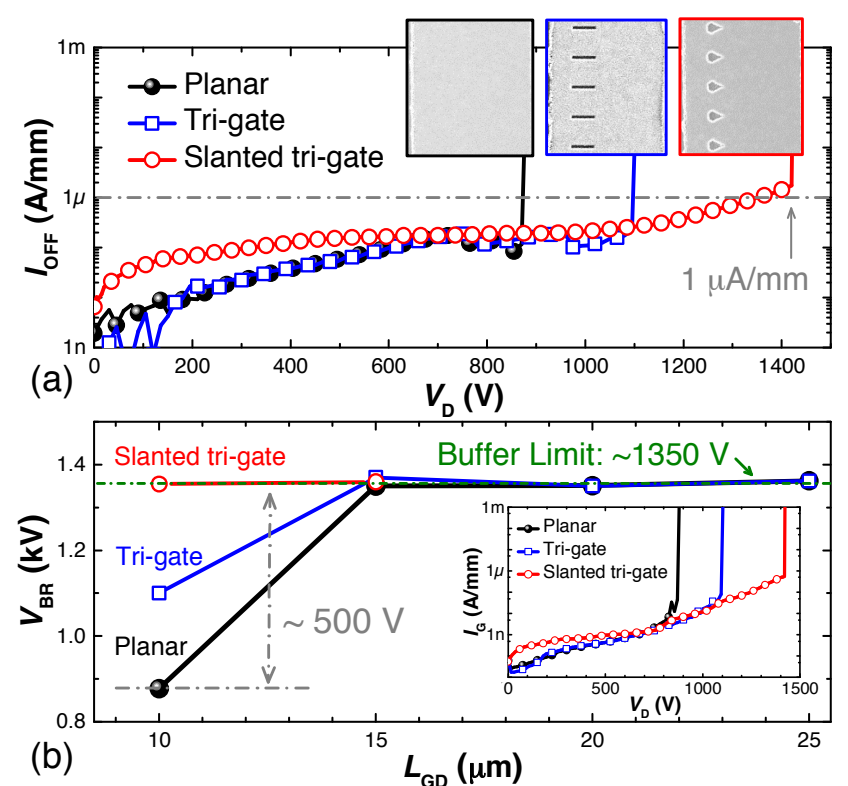

Fig. 4. (a) Typical breakdown characteristics of MOSHEMTs with planar gates, tri-gates and slanted tri-gates and (b) their $L_{\mathrm{GD}}$-dependent $V_{\mathrm{BR}}$ (at 1 $\mu \mathrm{A} / \mathrm{mm})$, measured at $V_{\mathrm{G}}$ of $-10 \mathrm{~V}$ with a floating substrate, in which the $I_{\mathrm{OFF}}$ for all devices was normalized by their footprint width. The insets in (a) show the top-view SEM images of the gate region of the three types of devices. The inset in (b) shows the gate leakage current of the devices. The gate-to-source distance $\left(L_{\mathrm{GS}}\right)$, gate length $\left(L_{\mathrm{G}}\right)$ and $L_{\mathrm{GD}}$ are $1.5 \mu \mathrm{m}, 2.5 \mu \mathrm{m}$, and $10 \mu \mathrm{m}$, respectively.

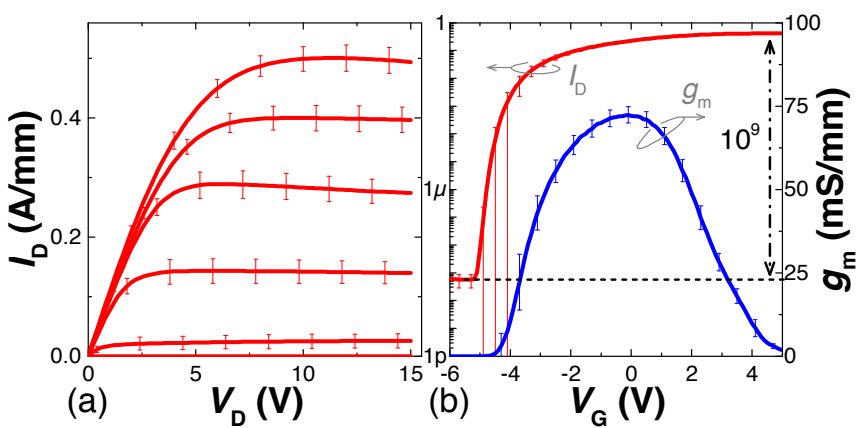

Fig. 5. (a) Average output and (b) transfer characteristics of the slanted tri-gate devices, normalized by their footprint width. The error bars were determined from measurements on six devices. The $L_{\mathrm{GS}}, L_{\mathrm{G}}$, and $L_{\mathrm{GD}}$ of all devices are 1.5 $\mu \mathrm{m}, 2.5 \mu \mathrm{m}$ and $10 \mu \mathrm{m}$, respectively.

and slanted tri-gate devices in Fig. 4(a). An improvement in $V_{\mathrm{BR}}$ from 877 to $1100 \mathrm{~V}$ at $1 \mu \mathrm{A} / \mathrm{mm}$ was observed with the introduction of the tri-gate region in a portion of the gate (as shown in the insets of Fig. 4(a)), which creates a region of a smaller $\left|V_{\mathrm{p}}\right|$ within the gate and converts the planar part of the gate towards the drain side into an effective FP, spreading more effectively the electric field as discussed previously (Fig. 2(b)). The $V_{\mathrm{BR}}$ was further improved with the slanted tri-gate to 1350 $\mathrm{V}$ at $1 \mu \mathrm{A} / \mathrm{mm}$. Figure 4(b) shows the large improvement in $V_{\mathrm{BR}}$ reaching the limit of our buffer layers with a $L_{\mathrm{GD}}$ as small as 10 $\mu \mathrm{m}$ due to the more effective spreading of the electric field by the slanted tri-gate. More importantly, the significant enhancement observed from the planar to the tri-gate and slanted tri-gate transistors was achieved by simply patterning the tri-gate and slanted tri-gate regions during the mesa etching to engineer the pinch-off voltage. The remainder of the device structure and fabrication process is exactly the same of the planar device. The larger $I_{\mathrm{OFF}}$ of the slanted tri-gate device at small voltages is likely due to its much smaller effective gate

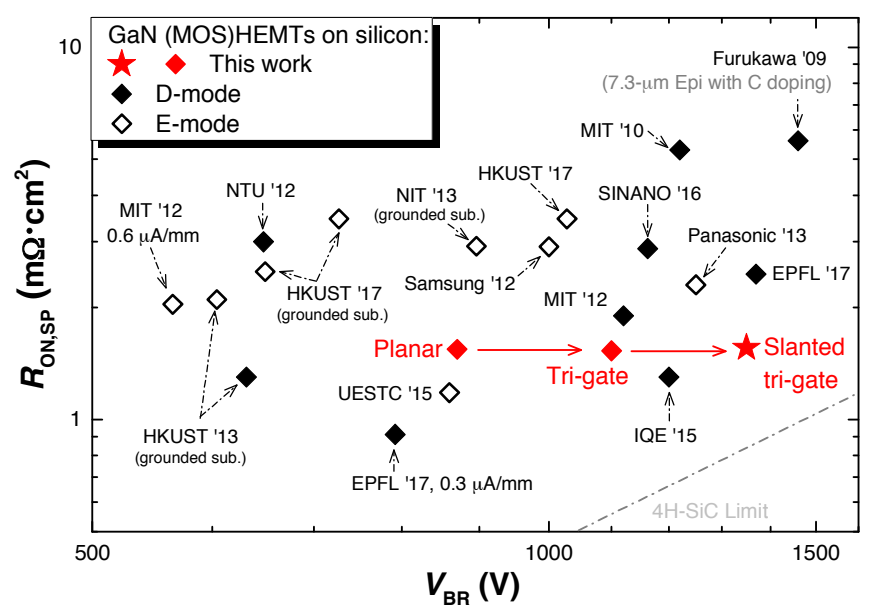

Fig. 6. Specific on-resistance $\left(R_{\mathrm{ON} . \mathrm{SP}}\right)$ versus $V_{\mathrm{BR}}$ benchmarks of the slanted tri-gate MOSHEMTs against state-of-the-art GaN E/D-mode (MOS)HEMTs on silicon by defining the $V_{\mathrm{BR}}$ at $I_{\mathrm{OFF}} \leq 1 \mu \mathrm{A} / \mathrm{mm}$. A $1.5-\mu \mathrm{m}$ transfer length for each ohmic contact was considered for calculation of the $R_{\mathrm{ON} \text { SP }}$, and the $V_{\mathrm{BR}}$ for all reference devices was re-calculated based on the reported data following the definition of $V_{\mathrm{BR}}$ at $I_{\mathrm{OFF}} \leq 1 \mu \mathrm{A} / \mathrm{mm}$.

length, since the three devices have the same buffer leakage current as they were fabricated on the same chip, and exhibited small gate leakage currents $\leq 2 \mathrm{nA} / \mathrm{mm}$ at $750 \mathrm{~V}$ (the inset of Fig. 4(b)). The $I_{\mathrm{OFF}}$ of the slanted tri-gate devices at high voltages was similar to the other devices, which shows that the larger $I_{\mathrm{OFF}}$ at low biases does not degrade the value of the slanted tri-gate in enhancing the $V_{\mathrm{BR}}$ of the device.

In addition to the high $V_{\mathrm{BR}}$, the slanted tri-gate device also exhibited excellent ON-state performance (Fig.5), presenting a high ON/OFF-current ratio over $10^{9}$ and a maximum $g_{\mathrm{m}}$ of 72.4 $\pm 2.4 \mathrm{mS} / \mathrm{mm}$ (at $V_{\mathrm{D}}$ of $5 \mathrm{~V}$ ), higher than in the planar device $(66.1 \pm 2 \mathrm{mS} / \mathrm{mm})$. The $R_{\mathrm{ON}}$ was $9.4 \pm 0.5 \Omega \cdot \mathrm{mm}$, very close to that of the planar device $(9.1 \pm 0.2 \Omega \cdot \mathrm{mm})$ despite the removal of carriers in its gate region during the nanowire etching.

The slanted tri-gate devices were benchmarked against state-of-the-art GaN (MOS)HEMTs on silicon substrates (Fig. $6)$. The high $V_{\mathrm{BR}}$ of $1350 \mathrm{~V}$ at $1 \mu \mathrm{A} / \mathrm{mm}$ presented in this work is comparable to the best-reported value of $V_{\mathrm{BR}}(1460 \mathrm{~V})$ [15] but with a $14-\mu \mathrm{m}$-smaller $L_{\mathrm{GD}}$, resulting in a $3.6 \mathrm{x}$-smaller $R_{\mathrm{ON}, \mathrm{SP}}$. The high-power FOM of the slanted tri-gate transistors was up to $1.2 \mathrm{GW} / \mathrm{cm}^{2}$, which is a record value to the best of our knowledge and reveals the significant potential of the slanted tri-gate for the enhancement of $V_{\mathrm{BR}}$ in $\mathrm{GaN}$ power devices while maintaining small $L_{\mathrm{GD}}$ and $R_{\mathrm{ON}}$.

\section{CONCLUSION}

In this work we demonstrated a novel slanted tri-gate architecture to enhance the voltage-blocking performance in lateral $\mathrm{GaN}$ devices, whose $V_{\mathrm{p}}$ can be tailored laterally by varying $w$ with lithography. This provides a new degree of freedom for engineering the distribution of electric field and opens enormous opportunities for nanostructured $\mathrm{GaN}$ devices in future power/RF applications.

\section{REFERENCES}

[1] R. Coffie, "Analytical Field Plate Model for Field Effect Transistors," IEEE Transactions on Electron Devices, vol. 61, no. 3, pp. 878-883, Mar. 2014. doi: 10.1109/TED.2014.2300115.

(C) 2017 IEEE. Personal use of this material is permitted. Permission from IEEE must be obtained for all other uses, in any current or future media, including reprinting/republishing this material for advertising or promotional purposes, creating new collective works, for resale or redistribution to servers or lists, or reuse of any copyrighted component of this work in other works. 
[2] R. Coffie, "Slant field plate model for field effect transistors," IEEE Trans. Electron Devices, vol. 61, pp. 2867-2872, Aug. 2014. doi: 10.1109/TED.2014.2329475.

[3] S. Karmalkar, M. S. Shur, G. Simin and M. A. Khan, "Field-plate engineering for HFETs," IEEE Trans. Electron Devices, vol. 52, pp. 2534-2540, Dec. 2005. doi: 10.1109/TED.2005.859568.

[4] S. Karmalkar and U. K. Mishra, "Enahncement of breakdown voltage in $\mathrm{AlGaN} / \mathrm{GaN}$ high electron mobility transistors using a field plate," IEEE Trans. Electron Devices, vol. 48, pp. 1515-1521, Aug. 2001. doi: $10.1109 / 16.936500$

[5] H. Xing, Y. Dora, A. Chini, S. Heikman, S. Keller and U. K. Mishra, "High breakdown voltage AlGaN-GaN HEMTs achieved by multiple field plates," IEEE Electron Device Lett., vol. 25, pp. 161-163, Apr. 2004. doi: 10.1109/LED.2004.824845

[6] V. Kumar, G. Chen, S. Guo, and I. Adesida, "Field-plated $0.25-\mu \mathrm{m}$ gate-length $\mathrm{AlGaN} / \mathrm{GaN}$ HEMTs with varying field-plate length," IEEE Trans. Electron Devices, vol. 53, pp. 1477-1480, Jun. 2006. doi: 10.1109/TED.2006.874090.

[7] Y. Dora, A. Chakraborty, L. McCarthy, S. Keller, S. P. Denbaars, and U. K. Mishra, "High breakdown voltage achieved on AlGaN/GaN HEMTs with integrated slant field plates," IEEE Electron Device Lett., vol. 27, pp. 713-715, Sept. 2006. doi: 10.1109/LED.2006.881020.

[8] Y. Pei, R. Chu, N. A. Fichtenbaum, Z. Chen, D. Brown, L. Shen, Stacia Keller, S. P. Denbaars, and U. K. Mishira, "Recessed slant gate AlGaN/GaN high electron mobility transistors with $20.9 \mathrm{~W} / \mathrm{mm}$ at 10 GHz,” Jpn. J. Appl. Phys., vol. 46, pp. L1089-L1089, Nov. 2007. doi: 10.1143/JJAP.46.L1087.

[9] C. S. Suh, Y. Dora, N. Fichtenbaum, L. McCarthy, S. Keller and U. K. Mishra, "High-Breakdown Enhancement-Mode AlGaN/GaN HEMTs with Integrated Slant Field-Plate," 2006 International Electron Devices Meeting, San Francisco, 2006,pp. 1-3. doi: 10.1109/IEDM.2006.346931.

[10] K. Kobayashi, S. Hatakeyama, T. Yoshida, Y. Yabe, D. Piedra, T. Palacios, T. Otsuji, and T. Suemitsu, "Improved breakdown voltage and $\mathrm{RF}$ characteristics in $\mathrm{AlGaN} / \mathrm{GaN}$ high-electron-mobility transistors achieved by slant field plates," Appl. Phys. Express, vol. 7, pp. 096501-1-096501-4, Aug. 2014. doi: 10.7567/APEX.7.096501.

[11] Y. Pei, Z. Chen, D. Brown, S. Keller, S. P. Denbaars, and U. K. Mishira, "Deep-submicrometer AlGaN/GaN HEMTs with slant field plate," IEEE Electron Device Lett. vol. 30, pp. 328-330, Apr. 2009. doi: 10.1109/LED.2009.2014790.

[12] T. Suemitsu, K. Kobayashi, S. Hatakeyama, N. Yasukawa, T. Yoshida, T. Otsuji, D. Piedra, and T. Palacios, "A new process approach for slant field plates in GaN-based high-electron-mobility transistors," Jpn. J. Appl. Phys., vol. 55, pp. 01AD02-1-01AD02-6, Nov. 2016. doi: 10.7567/JJAP.55.01AD02

[13] T. Deguchi, A. Kamada, M. Yamashita, H. Tomita, M. Arai, K. Yamasaki, and T. Egawa, "High-voltage AlGaN/GaN HFETs by using graded gate field plates," Electron. Lett., vol. 48, pp. 109-110, Jan. 2012. doi: 10.1049/el.2011.3171.

[14] J. Wong, K. Shinohara, A. L. Corrion, D. F. Brown, Z. Carlos, A. Williams, Y. Tang, J. F. Robinson, I. Khalaf, H. Fung, A. Schmitz, T. Oh, S. Kim, S. Chen, S. Burnham, A. Margomeos, and M. Micovic, "Novel Asymmetric Slant Field Plate Technology for High-Speed Low-Dynamic Ron E/D-mode GaN HEMTs," IEEE Electron Device Lett., vol. 38, pp. 95-98, Jan. 2017. doi: 10.1109/LED.2016.2634528.

[15] Nariaki Ikeda, Shusuke Kaya, Jiang Li, Takuya Kokawa, Mitsuru Masuda and Sadahiro Katoh, "High-power AlGaN/GaN MIS-HFETs with field-plates on Si substrates," 2009 21st International Symposium on Power Semiconductor Devices \& IC's, Barcelona, 2009, pp. 251-254. doi: 10.1109/ISPSD.2009.5158049.

[16] J. Ma and E. Matioli, "High performance tri-gate GaN power MOSHEMTs on silicon substrate," IEEE Electron Device Lett., vol. 38 pp. 367-370, Mar. 2017. doi: 10.1109/LED.2017.2661755.

[17] S. Liu, Y. Cai, G. Gu, J. Wang, C. Zeng, W. Shi, Z. Feng, H. Qin, Z. Cheng, K. J. Cheng and B. Zhang, "Enhancement-mode operation of nanochannel array (NCA) AlGaN/GaN HEMTs," IEEE Electron Device Lett. vol. 33, pp. 354-356, Jan. 2012. doi: 10.1109/LED.2011.2179003.

[18] J. Ma and E. Matioli, "High-voltage and low-leakage AlGaN/GaN tri-anode Schottky diodes with integrated tri-Gate transistors," IEEE Electron Device Lett., vol. 38, pp. 83-86, Jan. 2017. doi 10.1109/LED.2016.2632044

(C) 2017 IEEE. Personal use of this material is permitted. Permission from IEEE must be obtained for all other uses, in any current or future media, including reprinting/republishing this material for advertising or promotional purposes, creating new collective works, for resale or redistribution to servers or lists, or reuse of any copyrighted component of this work in other works. 